\title{
Giant orbital diamagnetism of three-dimensional Dirac electrons in $\mathrm{Sr}_{3} \mathrm{PbO}$ antiperovskite
}

\author{
S. Suetsugu $\odot,{ }^{1,2,3}$ K. Kitagawa, ${ }^{1}$ T. Kariyado, ${ }^{4}$ A. W. Rost,${ }^{2,5,6}$ J. Nuss, ${ }^{2}$ C. Mühle, ${ }^{2}$ M. Ogata $\odot,{ }^{1}$ and H. Takagi ${ }^{1,2,5}$ \\ ${ }^{1}$ Department of Physics, The University of Tokyo, 7-3-1 Hongo, Bunkyo-ku, Tokyo 113-0033, Japan \\ ${ }^{2}$ Max Planck Institute for Solid State Research, Heisenbergstrasse 1, 70569 Stuttgart, Germany \\ ${ }^{3}$ Department of Physics, Kyoto University, Kyoto 606-8502, Japan \\ ${ }^{4}$ International Center for Materials Nanoarchitectonics, National Institute for Materials Science, Tsukuba 305-0044, Japan \\ ${ }^{5}$ Institute for Functional Matter and Quantum Technologies, University of Stuttgart, Pfaffenwaldring 57, 70550 Stuttgart, Germany \\ ${ }^{6}$ SUPA, School of Physics and Astronomy, University of St Andrews, North Haugh, St. Andrews, Fife KY16 9SS, United Kingdom
}

(Received 30 December 2020; accepted 22 February 2021; published 10 March 2021)

\begin{abstract}
In Dirac semimetals, interband mixing has been known theoretically to give rise to a giant orbital diamagnetism when the Fermi level is close to the Dirac point. In $\mathrm{Bi}_{1-x} \mathrm{Sb}_{x}$ and other Dirac semimetals, an enhanced diamagnetism in the magnetic susceptibility $\chi$ has been observed and interpreted as a manifestation of such giant orbital diamagnetism. Experimentally proving their orbital origin, however, has remained challenging. The cubic antiperovskite $\mathrm{Sr}_{3} \mathrm{PbO}$ is a three-dimensional Dirac electron system and shows the giant diamagnetism in $\chi$ as in the other Dirac semimetals. ${ }^{207} \mathrm{~Pb}$ NMR measurements are conducted in this study to explore the microscopic origin of diamagnetism. From the analysis of the Knight shift $K$ as a function of $\chi$ and the relaxation rate $T_{1}^{-1}$ for samples with different hole densities, the spin and the orbital components in $K$ are successfully separated. The results establish that the enhanced diamagnetism in $\mathrm{Sr}_{3} \mathrm{PbO}$ originates from the orbital contribution of Dirac electrons, which is fully consistent with the theory of giant orbital diamagnetism.
\end{abstract}

DOI: 10.1103/PhysRevB.103.115117

\section{INTRODUCTION}

Dirac semimetals [1,2], whose band crossing is protected by the crystalline symmetry, have attracted considerable interest, largely because of the expected topological properties. The formation of unusual surface states is a direct consequence of the nontrivial topology of their band structure with Dirac dispersions [3]. The Berry curvature around the Dirac node gives rise to unconventional responses to magnetic fields such as a nontrivial phase shift in quantum oscillations [4,5] and a chiral anomaly [6,7]. So far, the research effort has focused mainly on such intraband Berry curvature and related physics. However, it may be tempting to note that interband effects should not be dismissed here; a nontrivial topology of band mixing between the conduction and the valence bands can lead to an interband Berry connection [8] and gives rise to exotic phenomena as in the case for the intraband topological effect. The giant orbital diamagnetism of Dirac electrons $[9,10]$ may be viewed as such an interband topological effect. This mechanism is distinct from the other kinds of magnetisms originating from itinerant electrons, Pauli paramagnetism and Landau diamagnetism, which are scaled by the density of states of itinerant electrons at the Fermi level.

Published by the American Physical Society under the terms of the Creative Commons Attribution 4.0 International license. Further distribution of this work must maintain attribution to the author(s) and the published article's title, journal citation, and DOI. Open access publication funded by the Max Planck Society.
A large diamagnetism of the order of $10^{-4} \mathrm{emu} / \mathrm{mol}$, larger than the expected Larmor core diamagnetism of $\sim$ $10^{-5} \mathrm{emu} / \mathrm{mol}$, was recognized long ago in the measurements of bulk magnetic susceptibility in semimetal Bi [11], which is known to host massive three-dimensional Dirac bands with a small number of electrons. The large diamagnetism of $\mathrm{Bi}$ was found to be further enhanced by Sb-doping in $\mathrm{Bi}_{1-x} \mathrm{Sb}_{x}$ [12,13], which reduces the number of electrons in the Dirac bands [14]. Eventually the diamagnetism is maximized when the Fermi level $E_{\mathrm{F}}$ lies in the Dirac massgap [Fig. 1(a)] with $\mathrm{Sb} x>x_{c}=0.07$. Recently discovered three-dimensional (3D) Dirac semimetals also show a large diamagnetism of similar magnitude in the bulk magnetic susceptibility [15-18], when their $E_{\mathrm{F}}$ is located near the Dirac points.

The microscopic origin of the giant diamagnetism in Dirac semimetals, in particular, $\mathrm{Bi}_{1-x} \mathrm{Sb}_{x}$, has been a subject of intense theoretical debate over decades. The early attempts to understand it as a Landau diamagnetism failed to explain the maximized diamagnetic response when $E_{\mathrm{F}}$ lies in the gap. The orbital magnetism of Dirac bands in the presence of interband effects was then proposed to be the origin [9], which can explain the enhancement of diamagnetism towards the Dirac point reasonably and has been accepted as the theoretical picture behind the giant diamagnetism. One of the intuitive pictures for the theoretical understanding of giant orbital diamagnetism is based on the $E$-linear density of states $D\left(E_{\perp}\right)$, where $E_{\perp}$ is a two-dimensional kinetic energy for the momentum perpendicular to the applied field. When Dirac electrons are confined in Landau levels under a magnetic field, the average energy gain and loss are not balanced, in contrast 
(a)

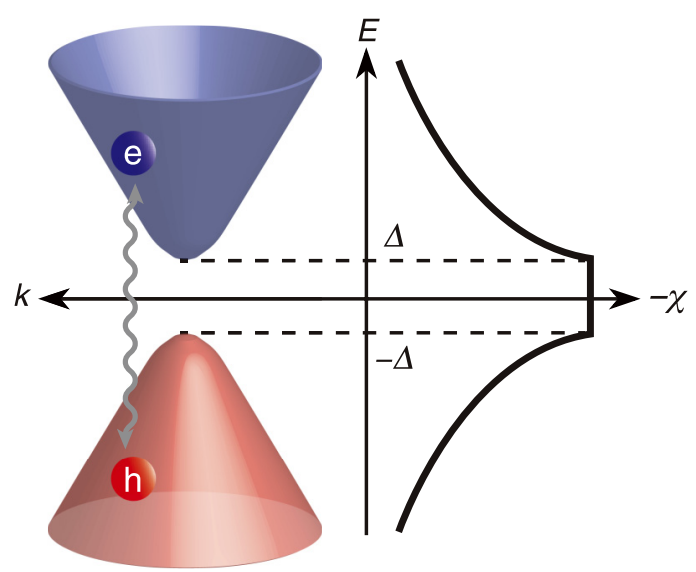

(b)

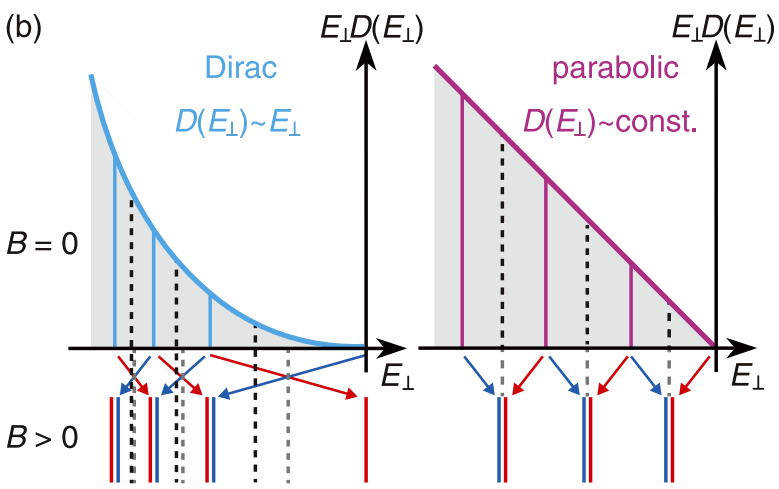

FIG. 1. Giant orbital diamagnetism of Dirac electrons. (a) Schematic energy dispersion of a massive Dirac electron band (left) and the expected giant orbital diamagnetism as a function of the Fermi level $E_{F}$ (right). $\Delta$ is a Dirac mass gap. The orbital diamagnetism takes a maximum with $E_{\mathrm{F}}$ in the Dirac mass gap. (b) Energy density $E_{\perp} D\left(E_{\perp}\right)$ as a function of $E_{\perp}$ for a Dirac band (left) and an ordinary parabolic band (right). $E_{\perp}$ and $D\left(E_{\perp}\right)$ represent the kinetic energy originating from the two-dimensional momentum perpendicular to the applied field and the density of states as a function of $E_{\perp}$, respectively. The Landau levels in a magnetic field with spin up and down are indicated by the blue and the red lines, respectively. The average $E_{\perp}$ of the Landau levels in a magnetic field, to which the gray shaded area of the zero field $D\left(E_{\perp}\right)$ condenses, is indicated by the gray dashed lines. They are larger than the average $E_{\perp}$ of the corresponding gray shaded area (black dashed line) for the Dirac band but identical to that for the normal parabolic band.

to the case for ordinary parabolic bands with constant $D\left(E_{\perp}\right)$ [10] [Fig. 1(b)], which increases the total free energy of Dirac bands under a magnetic field and gives rise to a diamagnetism. Note that the orbital diamagnetism comprises the contributions from all the electrons occupying the Dirac bands, not only from the electrons around the $E_{\mathrm{F}}$, as in the Pauli spin paramagnetism and the Landau (orbital) diamagnetism. This picture naturally explains the maximally enhanced orbital susceptibility when the $E_{\mathrm{F}}$ lies in the Dirac mass gap.

Despite the progress in the theoretical understanding of the origin of giant diamagnetism, its experimental verification has remained challenging, as it requires a separation of the orbital component from the spin component. The expected orbital diamagnetism from Dirac electrons of the order of $10^{-4} \mathrm{emu} / \mathrm{mol}$ is large but still could be comparable to the spin
Pauli paramagnetism, for example, when bands other than the Dirac bands contribute and/or the $g$ factor is enhanced from 2. Magnetic resonance techniques, in principle, could analyze the contributions from different origins. The microscopic magnetism of $\mathrm{Bi}_{1-x} \mathrm{Sb}_{x}$ has been studied by nuclear magnetic resonance (NMR) [19], muon spin rotation $(\mu \mathrm{SR})$ [20,21], perturbed angular distribution [22,23], and $\beta$-NMR [24]. The verification of the orbital character of diamagnetism using these techniques, however, has been far from complete. In the case of NMR, the large electric quadrupole interaction of a nuclear spin $I \geqslant 1$ in ${ }^{209} \mathrm{Bi}$ NMR has imposed critical constraints on the detailed analysis of the electronic contribution and the separation of spin and orbital contributions. An NMR study on an $I=1 / 2$ nuclear spin system, without electric quadrupole and phonon interactions, should be a promising approach to verify the orbital origin of the giant diamagnetism in Dirac semimetals. Dirac semimetals containing appropriate nuclear species, however, have been limited.

$\mathrm{Sr}_{3} \mathrm{PbO}$, the material we study here, is a member of the antiperovskite family $A_{3} T t \mathrm{O}(A=\mathrm{Ca}, \mathrm{Sr}, \mathrm{Ba}, \mathrm{Eu} ; T t=\mathrm{Si}$, $\mathrm{Ge}, \mathrm{Sn}, \mathrm{Pb}$ ) [25] and is theoretically proposed to be a 3D massive Dirac electron system [26,27] with topological surface states $[28,29]$. The cubic antiperovskite structure of $\mathrm{Sr}_{3} \mathrm{PbO}$ is shown in Fig. 2(a), where the $\mathrm{Pb}$ atoms are on the corners of cubic unit cell and the $\mathrm{Sr}$ atoms form an octahedron surrounding the $\mathrm{O}$ atom at the center. In the ionic limit, the valence states of constituent ions can be expressed as $\mathrm{Sr}^{2+}{ }_{3} \mathrm{~Pb}^{4-} \mathrm{O}^{2-}$. In the reported band structures [26], the valence and the conduction bands indeed consist of the fully occupied $6 p$ orbitals of $\mathrm{Pb}^{4-}$ and the empty $4 d$ orbitals of $\mathrm{Sr}^{2+}$, respectively. The $6 p$ and the $4 d$ bands overlap marginally and a gap opens almost everywhere on the band crossing plane. The $C_{4}$ rotational symmetry, however, protects the band crossing at six equivalent points on $\Gamma$-X lines [Fig. 2(b)], which leads to six moderately anisotropic 3D Dirac bands free from the other parabolic bands. The 3D Dirac band has a very small mass gap of $\sim 10 \mathrm{meV}$, which is created by the admixture of higher energy orbital states via spin-orbit coupling. The six Dirac bands merge at $-125 \mathrm{meV}$ below the Dirac points, giving rise to a saddle point. Below the saddle point, multiband Fermi surfaces are expected to emerge when the Fermi level lies in this region. This region is essentially away from the Dirac physics. The presence of 3D Dirac electrons in $\mathrm{Sr}_{3} \mathrm{PbO}$ is supported by recent experiments $[18,30]$, which show the presence of extremely light mass $\left(\sim 0.01 m_{\mathrm{e}}\right)$ holes and $B$-linear magnetoresistance. Angle-resolved photoemission spectroscopy on a sister compound $\mathrm{Ca}_{3} \mathrm{PbO}$ confirms the Dirac dispersion of the valence band predicted by band calculations [31].

The $\mathrm{Pb}$ antiperovskite should provide a promising arena for NMR studies of Dirac semimetals to verify the orbital character of the giant diamagnetism from Dirac electrons, as ${ }^{207} \mathrm{~Pb}$ hosts $I=1 / 2$ nuclear moment in contrast to ${ }^{209} \mathrm{Bi}$. Here, we report ${ }^{207} \mathrm{~Pb}$ NMR and magnetic susceptibility $\chi$ studies of the 3D Dirac system $\mathrm{Sr}_{3} \mathrm{PbO}$. An enhanced diamagnetism is observed in the magnetic susceptibility $\chi$ as in $\mathrm{Bi}$ and other 3D Dirac systems. Using the Korringa relation with the spinlattice relaxation rate $T_{1}^{-1}$, we show that the spin contribution $K_{\text {spin }}$ in $K$ cannot account for the enhanced diamagnetism. The $K-\chi$ plot can be analyzed as the superposition of the spin and the orbital contributions with distinct hyperfine coupling 
(a)

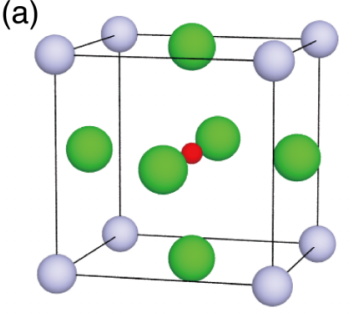

(b)

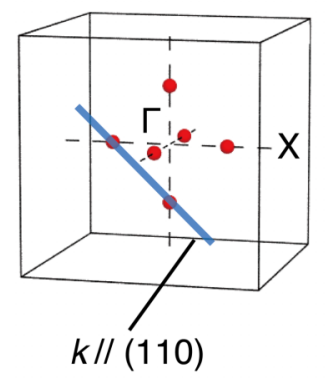

(c)

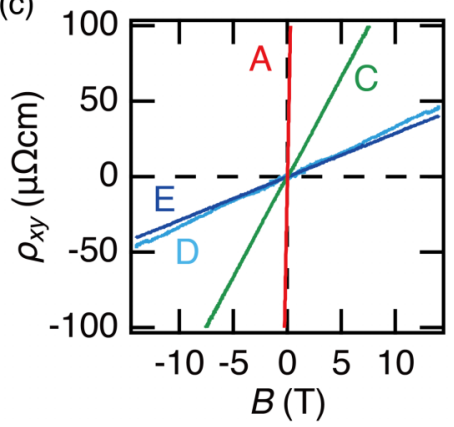

(d)
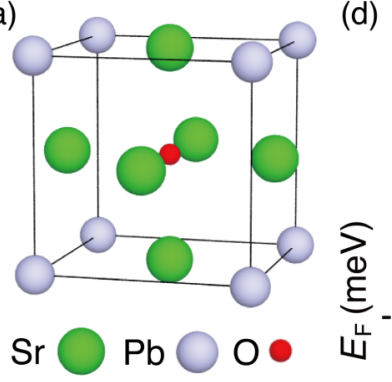

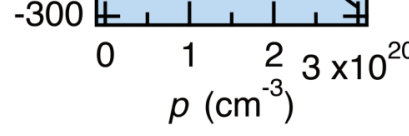

(f)
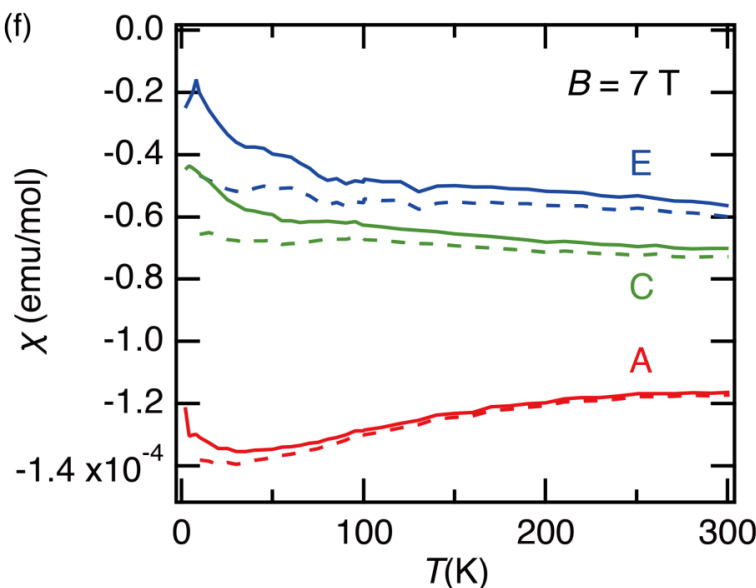

FIG. 2. Basic electronic structure and bulk magnetic susceptibility in $\mathrm{Sr}_{3} \mathrm{PbO}$ antiperovskite. (a) and (b) Crystal structure and the first Brillouin zone for $\mathrm{Sr}_{3} \mathrm{PbO}$ antiperovskite. Six Dirac points [red points in (b)] are protected by $C_{4}$ rotational symmetry along $\Gamma$-X lines. The blue line parallel to the (110) direction connects two Dirac points, between which a saddle point (SP) exists. (c) Field dependence of Hall resistivity $\rho_{x y}$ in the zero-field limit. The slopes yield the hole densities $1.6 \times 10^{18}, 5.0 \times 10^{19}, 2.0 \times 10^{20}$ and $2.2 \times 10^{20} \mathrm{~cm}^{-3}$ for samples $\mathrm{A}, \mathrm{C}, \mathrm{D}$, and $\mathrm{E}$, respectively. (d) Total carrier density as a function of the Fermi energy $\left(E_{F}\right)$ obtained from a band calculation, which gives the estimates of $E_{F}$ (dashed lines) for samples A, C, D, and $\mathrm{E}$ as $-45,-125,-235$, and $-250 \mathrm{meV}$, respectively, from the experimental hole densities obtained from (c). (e) Schematic band structure of $\mathrm{Sr}_{3} \mathrm{PbO}$ antiperovskite for a $k$ axis along the blue line in (b), which is divided into three regimes, the Dirac bands (red area), the saddle point (SP) and the multibands (blue area). The Dirac bands enclosed by the gray dashed rectangle give rise to the giant orbital diamagnetism as illustrated in Fig. 1(a). (f) Temperature dependence of magnetic susceptibility $\chi$ for samples A, C, and E (solid lines). The magnitude of diamagnetic susceptibility increases with decreasing the hole density $p$, in particular very rapidly from sample $\mathrm{C}$ to $\mathrm{A}$. By subtracting Curie-like contributions at low temperatures, the intrinsic behaviors of $\chi$ are estimated (dashed lines).

constants, consistently with the analysis of the Korringa relation. The estimated orbital hyperfine constant indicates the delocalized nature of electrons in charge of the large orbital susceptibility. These results strongly affirm that the enhanced diamagnetism originates from the giant orbital susceptibility of Dirac electrons.

\section{METHOD}

Five batches of $1 \mathrm{~g}$ of polycrystalline $\mathrm{Sr}_{3} \mathrm{PbO}$, A-E, were synthesized from $\mathrm{PbO}$ and 3-5\% excess amount of $\mathrm{Sr}$ metal from the stoichiometry in a sealed tantalum ampoule, as reported previously [25]. All the syntheses and the measure- ments were conducted without exposing the samples to air because the samples are extremely air sensitive.

The magnetization of the powder samples of $\mathrm{Sr}_{3} \mathrm{PbO}$ was measured by a commercial superconducting quantum interference device (SQUID) magnetometer (Quantum Design MPMS) with an applied magnetic field of $7 \mathrm{~T}$. The powder samples were sealed in a glass tube with a small amount of helium gas during the measurements. The magnetization of the glass tube without the sample was measured separately. The background correction was conducted by subtracting the scanning pattern of the SQUID signal without the sample from that with the sample. The magnetization of the sample was then calculated by fitting the scanning pattern of the SQUID signal after the subtraction of the background. The Curie-like 
contribution from the impurities at low temperatures was estimated by a fitting of the magnetization $M(T)$ at $7 \mathrm{~T}$ between 10 and $50 \mathrm{~K}$ with $M=M_{0}+C / T . M_{0}$ is a constant, $T$ is the temperature and $C$ is a fitting parameter.

The ${ }^{207} \mathrm{~Pb}$ NMR measurements were conducted using a Carr-Purcell-Meiboom-Gill multiecho sequence with 10 echoes for samples A-C and a conventional spin-echo sequence for samples D and E. The powder samples were immersed in a high-quality mineral oil, sealed in straws with epoxy resin inside an argon-filled glove box, and then mounted in an NMR coil. The magnetic field $B \sim 7 \mathrm{~T}$ was calibrated by the ${ }^{63} \mathrm{Cu}$ NMR spectrum of the coil using a gyromagnetic ratio $\gamma / 2 \pi=11.2893305 \mathrm{MHz} / \mathrm{T}$ and Knight shift $K=0.200 \%$. NMR spectra were obtained by a combination of Fourier transformation and frequency sweep (Fourier-Step-Sum technique). The origin of the peak shift $\Delta f$ is defined as $\gamma B$ with a gyromagnetic ratio of ${ }^{207} \mathrm{~Pb} \gamma / 2 \pi=$ $8.9072353 \mathrm{MHz} / \mathrm{T}$ [32]. The NMR Knight shift $K=\Delta f / \gamma B$ was determined from the NMR frequency shift of the $I=1 / 2$ nucleus. The spin-lattice relaxation rate $T_{1}{ }^{-1}$ was derived from a single-exponential fit of a relaxation curve collected by a standard inverse saturation recovery method.

The density functional theory (DFT) calculation was performed using the QUANTUM ESPRESSO package $[33,34]$ with the relativistic PAW dataset, setting the wave function and charge density cutoffs to 50 and $400 \mathrm{Ry}$, respectively. The maximally localized Wannier functions (MLWF) are derived using the WANNIER90 package [35]. We put $s$ - and $p_{x, y, z}$-like orbitals on the $\mathrm{Pb}$ site and $d_{x^{2}-y^{2}, z x, y z}$-like orbitals on the three $\mathrm{Sr}$ sites in the unit cell as the initial guess functions. Together with the spin degrees of freedom, this forms a 26-band model [(one $s$ orbital, three $p$ orbitals, and $3 \times 3 d$ orbitals $) \times(2$ spins)]. We set $-3.3 \mathrm{eV}$ to $1.486 \mathrm{eV}$ (measured from $E_{F}$ ) as a frozen energy window, i.e., the spectral features within this energy range are faithful to the DFT results up to minor errors caused by limited number of momentum sampling points.

The effective model used in our analysis is a tight-binding model whose basis orbitals are MLWFs derived from the DFT, which allows us to incorporate the saddle points in the band structure into the following calculation of magnetic susceptibility.

In the calculation of magnetic susceptibility, we start from the Hamiltonian $H=H_{\text {kin }}+H_{1}$ with

$$
\begin{gathered}
H_{\text {kin }}=\sum_{i a \sigma, j b \sigma^{\prime}} t_{i a \sigma, j b \sigma^{\prime}} c_{i a \sigma}^{\dagger} c_{j b \sigma^{\prime}}, \\
H_{1}=\mu_{B} \sum_{i a}\left(2 S_{i}^{z}+L_{i a}^{z}\right) B_{z} .
\end{gathered}
$$

The hopping integrals in $H_{\text {kin }}$ are from the MLWF method with the Peierls phase added by hand for the magnetic field. $L^{z}$ in $H_{1}$ is the $z$ component of the (local) angular momentum, $l=1$ for $\mathrm{Pb}-6 p$ orbitals and $l=2$ for $\mathrm{Sr}-4 d$ orbitals.

We can derive the magnetic susceptibility by expanding the Green's function for $H$ as a series of $B_{z}$, and there is a prescription to carry out this expansion in a gauge invariant manner [36]. Without $H_{1}$, the formula has been derived in
Refs. [37,38]. Adapting the techniques in Refs. [36,38] to include $H_{1}$, we end up with the formulas

$$
\begin{gathered}
\chi=\chi_{\text {orbital }}+\chi_{\text {cross }}+\chi_{\text {spin }}, \\
\chi_{\text {orbital }}=\frac{e^{2}}{4 \hbar^{2}} \widetilde{\operatorname{Tr}}\left(\Sigma_{x y}+\gamma_{x y}\right) g \Sigma_{x y} g+(x \leftrightarrow y), \\
\chi_{\text {cross }}=i \frac{\mu_{B} e}{\hbar} \widetilde{\operatorname{Tr}} M g\left(\Sigma_{x y}-\Sigma_{y x}\right) g, \\
\chi_{\text {spin }}=-\mu_{B}^{2} \widetilde{\operatorname{Tr}} M g M g .
\end{gathered}
$$

The contributions from $\chi_{\text {cross }}$ and $\chi_{\text {spin }}$ are absent without $H_{1}$. Here, $\widetilde{T r}$ is a shorthand notation of $\frac{T}{N} \sum_{\omega_{n}, k} \operatorname{Tr}, g=$ $\left(i \omega_{n}-H_{k}\right)^{-1}$ with $H_{k}$ being the Fourier-transformed $H_{\text {kin }}$ without the Peierls phase, $M=2 S_{z}+L_{z}$, and $\Sigma_{\alpha \beta}=\gamma_{\alpha} g \gamma_{\beta}$. $\gamma_{\mu}\left(\gamma_{\mu \nu}\right)$ is defined as $\gamma_{\mu}=\nabla_{\mu} H_{k}\left(\gamma_{\mu \nu}=\nabla_{\mu} \nabla_{\nu} H_{k}\right)$, where $\nabla_{\mu} X=\frac{\partial X}{\partial k_{\mu}}+i\left[\hat{\epsilon}_{\mu}, X\right]$ with $\left(\hat{\epsilon}_{\mu}\right)_{a b}=\delta_{a b} \epsilon_{a \mu}$, where $\epsilon_{a \mu}$ is the $\mu$ component of the position of orbital $a$ in a unit cell.

\section{RESULTS AND DISCUSSION}

\section{A. Carrier density and Fermi level}

Five polycrystalline samples of $\mathrm{Sr}_{3} \mathrm{PbO}$ from different batches A-E with different hole densities $(p)$ from $\sim 10^{18}$ to $\sim 10^{20} \mathrm{~cm}^{-3}$ were investigated. The Hall resistivity $\rho_{x y}$ in the zero-field limit gives positive Hall coefficients $R_{H}=+3.8$, 0.13, 0.032, and 0.029 and $\mathrm{cm}^{-3} / \mathrm{C}$ [Fig. 2(c)], yielding hole densities $p=1.6 \times 10^{18}, 5.0 \times 10^{19}, 2.0 \times 10^{20}$, and $2.2 \times$ $10^{20} \mathrm{~cm}^{-3}$ for samples A, C, D, and E, respectively. Sample B should have a comparable but only slightly smaller $p$ than sample $\mathrm{C}$, judging from the NMR data. The donors very likely correspond to $0.01-1 \%$ level of cation defects and/or excess oxygens, which are introduced partially to relax the extremely reduced anionic state of $\mathrm{Pb}^{4-}$. The result of band calculations in Fig. 2(d) indicates that the experimentally observed hole densities correspond to $E_{F}$ being $-45,-125,-235$, and $-250 \mathrm{meV}$ (measured from the center of the mass gap) for samples A, C, D, and E, respectively. These $E_{F} \mathrm{~s}$ are indicated by the dashed lines in the schematic band picture shown in Fig. 2(e). The $E_{F}$ of sample A lies in the Dirac band region while that of sample $C$ (and $B$ ) is around the saddle point and those of samples D and $\mathrm{E}$ are in the multi-Fermi surface region below the saddle point. The Dirac physics should manifest itself almost exclusively in sample A.

\section{B. Bulk magnetic susceptibility}

The magnetic susceptibilities $\chi(T)$ for three samples A, $\mathrm{C}$, and E, shown in Fig. 2(f), are found to be all diamagnetic. The magnitude of diamagnetism increases with decreasing the hole concentration $p$ and hence increasing $E_{F}$ from $\mathrm{E}$ to A. The increase from samples $\mathrm{C}$ and $\mathrm{E}$ to sample $\mathrm{A}$ with $E_{F}$ in the Dirac bands is particularly significant and as large as of the order of $10^{-4} \mathrm{emu} / \mathrm{mol}$, which is comparable to the large diamagnetism observed in other Dirac semimetals $[15,16]$. An appreciable temperature dependence is observed particularly for sample A. The $\chi(T)$ of sample A shows a clear decrease with lowering temperature to $\sim 30 \mathrm{~K}$, which should be an intrinsic behavior of magnetic susceptibility. This is followed 
(a)
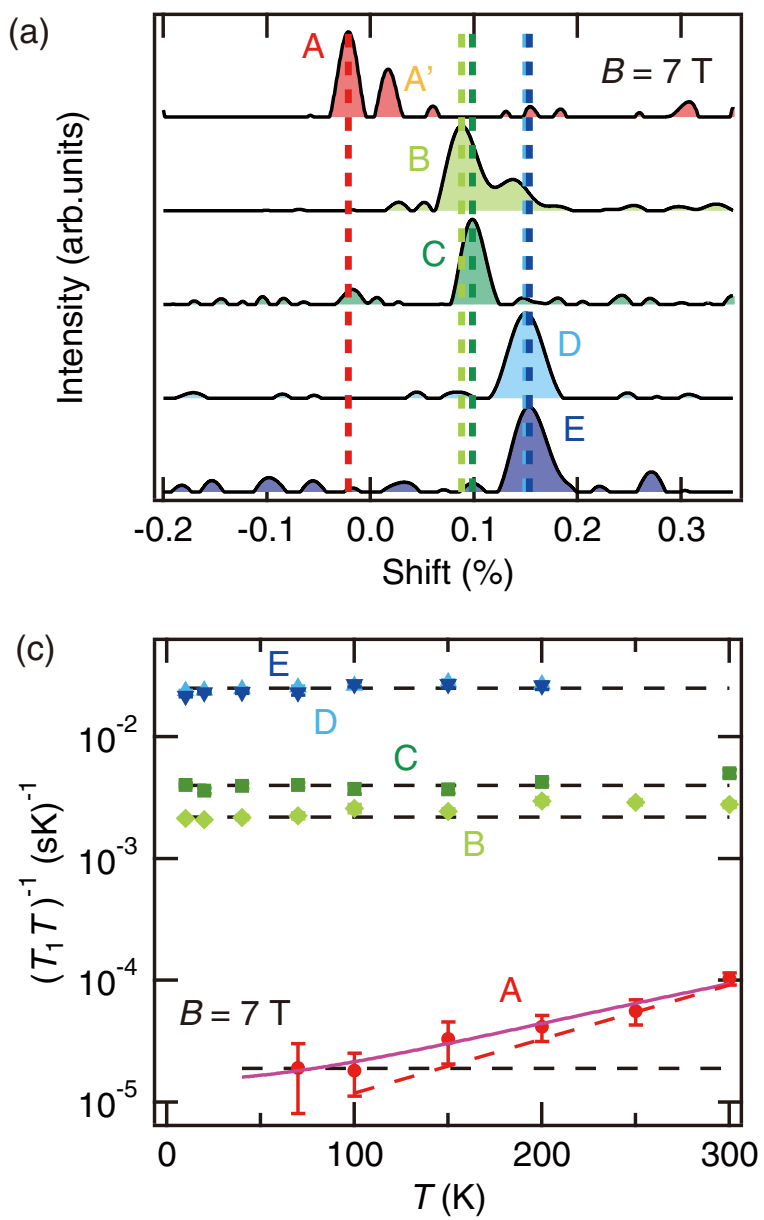
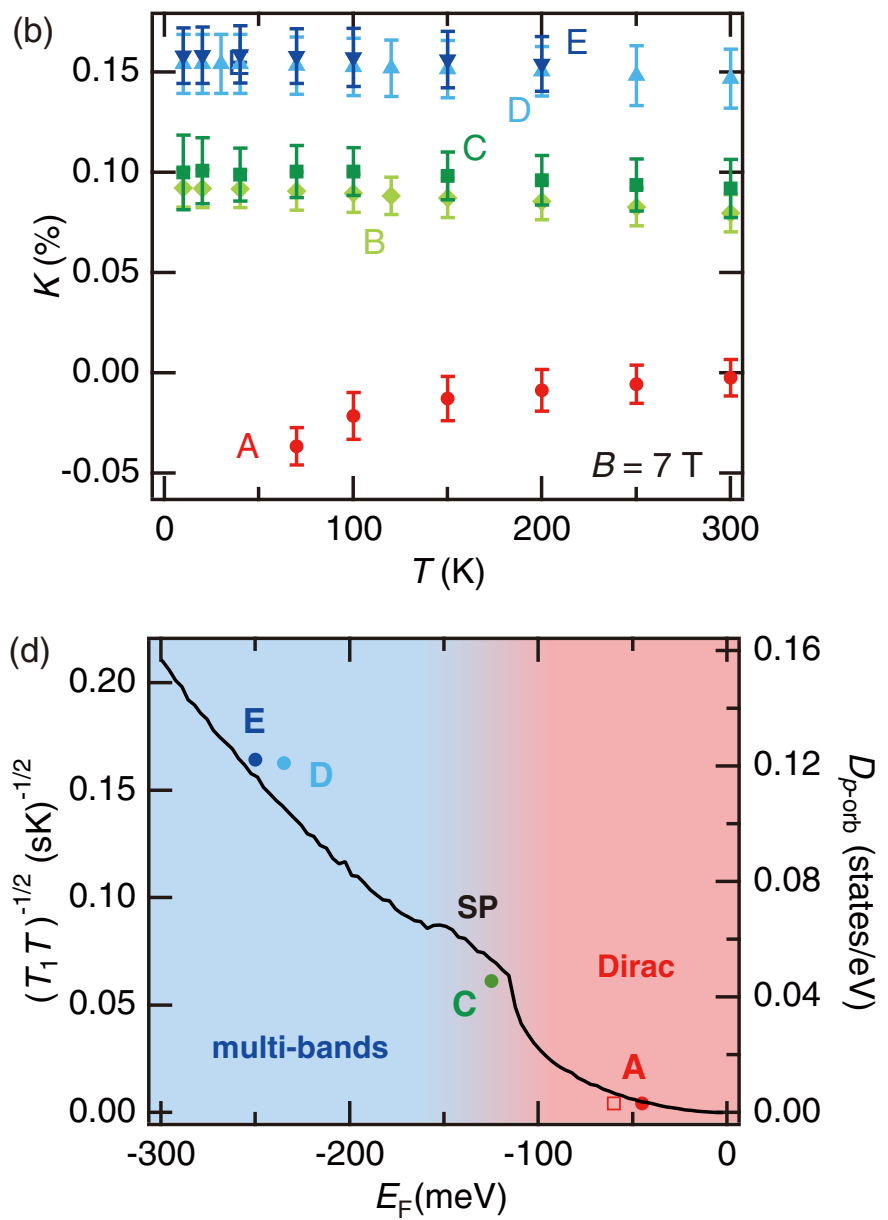

FIG. 3. NMR spectra, Knight shift $K$ and spin lattice relaxation rate $T_{1}^{-1}$ in $\mathrm{Sr}_{3} \mathrm{PbO}$ antiperovskite. (a) NMR spectra for samples A-E at a temperature $T=150 \mathrm{~K}$. The peak positions systematically shift to the negative side upon decreasing the hole density $p$. The hole density $p$ and the Fermi level $E_{F}$ for each sample are displayed in Figs. 2(d) and 2(e). (b) Temperature dependence of the NMR Knight shift $K(T)$ for samples A-E. Note the positive correlation between $K(T)$ and $\chi(T)$ in Fig. 2(f) is indicative of the positive hyperfine coupling constants $A_{\text {spin }}$ and $A_{\text {orb }}$. (c) Temperature dependencies of the spin-lattice relaxation rate divided by temperature $\left(T_{1} T\right)^{-1}(T)$ for samples A-E. The Korringa law, $\left(T_{1} T\right)^{-1}=$ constant (black dashed line) holds well. A crossover from $T$ independence to $T^{2}$ dependence (red dashed line) for sample A reflects the strongly energy dependent density of states of Dirac electrons. (d) Fermi energy $E_{F}$ dependence of $\left(T_{1} T\right)^{-1 / 2}$ at $100 \mathrm{~K}(\mathrm{closed}$ circles), which is scaled well with the calculated partial density of states for $\mathrm{Pb} 6 p$ orbitals at $E_{F}, D_{p \text {-orb }}$ (black line).

by a Curie-like increase very likely associated with magnetic impurities $(0.01 \%$ level of $s=1 / 2$ impurities) at low temperatures. The other samples with a high hole density $\left(E_{F}\right)$ show a monotonic increase of $\chi(T)$ from room temperature down to $2 \mathrm{~K}$, with a clear Curie-like behavior of similar magnitude as sample A at low temperatures. It is not possible, however, to fit the $\chi(T)$ behavior for samples $\mathrm{C}$ and $\mathrm{E}$ over entire temperature range only with a Curie-Weiss contribution and a constant offset, particularly at high temperatures above 100 $\mathrm{K}$. This indicates the presence of very weak but appreciable increase of the intrinsic $\chi(T)$ [dashed lines in Fig. 2(f)] with lowering $T$ in samples $\mathrm{C}$ and $\mathrm{E}$ at least at high temperatures above $100 \mathrm{~K}$ where the Curie contribution is negligibly small. Note that the weak temperature dependence of the intrinsic $\chi(T)$ for samples $\mathrm{C}$ and $\mathrm{E}$ is negative, opposite to that of sample A.

The enhanced diamagnetism in sample A with the $E_{F}$ in the Dirac bands should represent the same large diamagnetism observed in $\mathrm{Bi}_{1-x} \mathrm{Sb}_{x}$ and other Dirac semimetals, which cannot be described naively by the conventional kinds of magnetisms. The core diamagnetism is estimated to be $-8.5 \times$ $10^{-5} \mathrm{emu} / \mathrm{mol}$ for $\mathrm{Sr}_{3} \mathrm{PbO}$ [39], which should not depend appreciably on the $1 \%$ level of cation defects or excess oxygens. The Pauli paramagnetism calculated from the density of states in the band calculation is only of the order of $10^{-5} \mathrm{emu} / \mathrm{mol}$ assuming $p \sim 2 \times 10^{20} \mathrm{~cm}^{-3}$ and $g=2$ for sample E, not as large as the difference of susceptibilities between samples A and E. At this point, however, the possibility of an enhanced $g$-factor, which could account for the difference, cannot be excluded completely.

\section{Giant diamagnetism in NMR Knight shift}

${ }^{207} \mathrm{~Pb}$ NMR measurements for samples A-E were conducted to verify the orbital origin of giant diamagnetism experimentally. The NMR spectra at $150 \mathrm{~K}$ are shown in Fig. 3(a). A systematic shift of the peak position as a function of $p$ (and hence $E_{F}$ ) is observed from sample A to E, implying 
that the NMR peaks originate from the bulk $\mathrm{Sr}_{3} \mathrm{PbO}$. We note that the observed shifts are different from those of possible impurity phases such as $1.081 \%,-0.034 \%$ and $0.444 \%$ for metallic $\mathrm{Pb}, \mathrm{PbO}$, and $\mathrm{PbO}_{2}$ [40], respectively. The presence of subpeaks in samples A and B are attributed to inhomogeneity/phase separation where small region(s) with a slightly different hole density from the main phase coexists (see also Fig. S3 in the Supplemental Material [41]).

The $p$ and $T$ dependence of the NMR Knight shift $K(T)$ [Fig. 3(b)], determined from the NMR spectra, is intimately related to those of the bulk magnetic susceptibility $\chi(T)$ shown in Fig. 2(f). $K(T)$ decreases with decreasing $p$ from sample $\mathrm{E}$ to sample A, particularly from sample $\mathrm{B}$ and $\mathrm{C}$ to sample A. With increasing temperature, $K(T)$ for sample A shows an appreciable increase while samples E-B show a very weak decrease of $K(T)$ in parallel to those of $\chi(T)$. The close correlation between $K(T)$ and $\chi(T)$ indicates that $K(T)$ captures the $p$ and $T$ dependence of $\chi(T)$ including the enhanced diamagnetism. The separation of spin and orbital contributions in $K(T)$ should provide a clue to identify the orbital origin of enhanced diamagnetism. Note that the observed results on powder samples should not be affected by a random orientation of grains because of the cubic crystal structure of $\mathrm{Sr}_{3} \mathrm{PbO}$.

The NMR Knight shift $K$ is comprised of several contributions as $K(T)=K_{\text {chem }}+K_{\text {spin }}(T)+K_{\text {orb }}(T)$, essentially the same as the bulk magnetic susceptibility. $K_{\text {spin }}$ and $K_{\text {orb }}$ are spin and orbital contributions, respectively. Each term is proportional to the respective susceptibilities $\chi_{\text {spin }}$ and $\chi_{\text {orb }}$ via the respective hyperfine coupling constants $A_{\text {spin }}$ and $A_{\text {orb }}$ as $K_{\text {spin }}=A_{\text {spin }} \chi_{\text {spin }} / N_{A} \mu_{B}$ and $K_{\text {orb }}=A_{\text {orb }} \chi_{\text {orb }} / N_{\mathrm{A}} \mu_{B}$. Here, $N_{\mathrm{A}}$ and $\mu_{B}$ are the Avogadro constant and the Bohr magneton, respectively (hereinafter we omit $N_{\mathrm{A}} \mu_{B}$ ). $A_{\text {spin }}$ and $A_{\text {orb }}$ are, in principle, different. The chemical shift $K_{\text {chem }}$ does not depend on $T$ and $p$ and gives a constant offset to $K$. The relationship between $K(T)$ and $\chi(T)$ for samples A, C, and E, the $K-\chi$ plot, is shown in Fig. 4(a), confirming the close correlation between $K(T)$ and $\chi(T)$. To exclude the influence from the extrinsic Curie contribution, we plot here data only for $T>$ $50 \mathrm{~K}$. The $K(T)-\chi(T)$ relationship for each sample is linear with almost common slopes among different samples. These straight lines for different samples, however, shift upward upon going from samples $\mathrm{A}$ to $\mathrm{E}$ with decreasing $E_{F}$ and do not fall onto a universal $K(T)-\chi(T)$ line, which strongly suggests that both $\chi_{\text {spin }}$ and $\chi_{\text {orb }}$ give appreciable contributions to the observed $K(T)$ with distinct $A_{\text {spin }}$ and $A_{\text {orb }}$. The $T$ dependence of $K(T)$ is highly likely dominated by one of the two contributions, as the $K(T)-\chi(T)$ slope originating from the $T$ dependence is universal. A correlation between $K(T)$ and $\chi(T)$ that is always positive implies that both $A_{\text {spin }}$ and $A_{\text {orb }}$ are positive.

\section{Korringa behavior in NMR spin lattice relaxation rate}

The Korringa behavior of the spin-lattice relaxation rate $T_{1}^{-1},\left(T_{1} T\right)^{-1}=$ constant, is observed for all samples A-E over a wide temperature range as shown in Fig. 3(c), which provides us with important hints to estimate $K_{\text {spin }}$ and hence $K_{\text {orb. }}$. The magnitude of $\left(T_{1} T\right)^{-1}$, which is proportional to
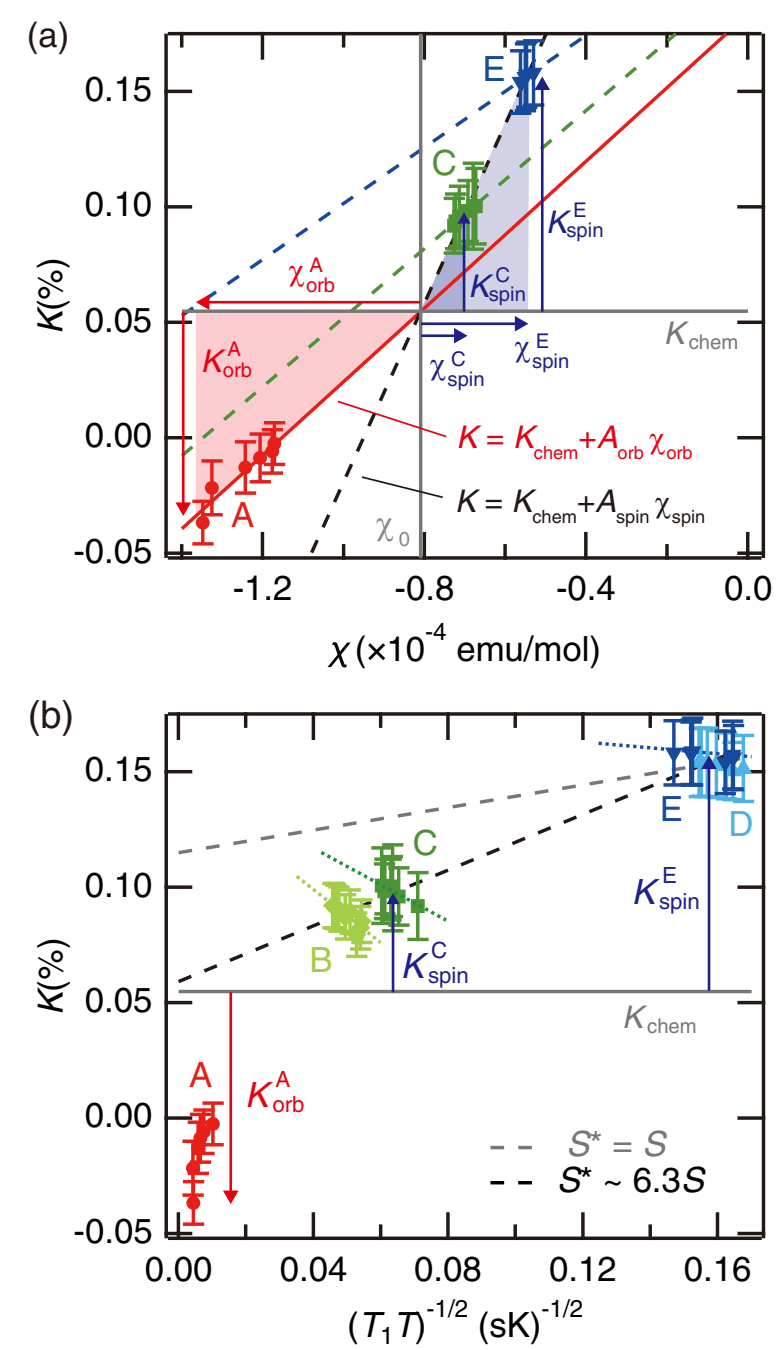

FIG. 4. Separation of Knight shift $K(T)$ into the spin and the orbital contributions. (a) Knight shift $K(T)$ vs magnetic susceptibility $\chi(T)$ plot for samples A, C, and E. The hole density $p$ and the Fermi level $E_{F}$ for each sample are displayed in Figs. 2(d) and 2(e). Here the intrinsic $\chi(T)$ after the subtraction of the Curie contribution [dashed line in Fig. 2(f)] was used for the plot. The orbital hyperfine coupling constant $A_{\text {orb }}=88 \pm 14 \mathrm{kOe} / \mu_{B}$ can be estimated from a linear fit to the $K(T)-\chi(T)$ relationship for sample A (red line), where the spin contribution $K_{\text {spin }}$ is almost zero. An upward deviation for samples $\mathrm{C}$ and $\mathrm{E}$ from the red line can be attributed to $K_{\text {spin. }}$. The black dashed line indicates the estimated $K_{\text {spin }}$ with the assumption of $K_{\text {orb }} \sim 0$. The crossing point between the red line and the black dashed line represents the chemical shift $K_{\text {chem }}$ and the core magnetic susceptibility $\chi_{0}$. (See the main text.) (b) Knight shift $K(T)$ as a function of the spin-lattice relaxation rate $\left(T_{1} T\right)^{-1 / 2}$ for sample $\mathrm{E}$ to sample A. The spin contribution $K_{\text {spin }}$ expected from the Korringa relationship $T_{1} T K_{\text {spin }}{ }^{2}=S$ is indicated by the gray dashed line. The black dashed line indicates a modified Korringa relationship with an enhanced Korringa constant $S^{*} \sim 6.3 S$, which assumes a dominant spin contribution and hence almost zero orbital contribution, $K_{\text {orb }} \sim$ 0 , for heavily doped samples B-E. Note that the extrapolation of the black dashed line to $\left(T_{1} T\right)^{-1 / 2}=0$ gives an estimate of the chemical shift $K_{\text {chem }}$ with the assumption of $K_{\text {orb }} \sim 0$ for samples B-E. The large and additional negative shift of sample A should be ascribed to the orbital contribution, $K_{\text {orb }}$. 
the square of the electron density of states $D\left(E_{F}\right)$ at $E_{F}$, increases systematically with increasing $p$ (decreasing $E_{F}$ ) from samples A to $\mathrm{E}$, which can be reasonably understood as the increase of $D\left(E_{F}\right)$. Indeed, as seen in Fig. 3(d), $\left(T_{1} T\right)^{-1 / 2}$ at $100 \mathrm{~K}$ for the samples with different $E_{F}$ are scaled well with the calculated $D\left(E_{F}\right)$ for the $6 p$ orbitals of $\mathrm{Pb}$, implying that $\left(T_{1} T\right)^{-1 / 2}$ captures the $D\left(E_{F}\right)$ that determines the spin contribution in $\chi(T)$ and $K(T)$. For sample A with the lowest hole density, a clear upward deviation from the Korringa behavior can be seen at high temperatures, which can be reasonably ascribed to the $T$ dependence of the thermally averaged density of states around $E_{F},\left\langle D(E)^{2}\right\rangle_{T}$ [42-45]. Assuming strongly $E$-dependent $D(E) \propto\left(E-E_{\mathrm{DP}}\right)^{2}$ around the Dirac points at $E=E_{\mathrm{DP}}$, the $T$ dependence of $T_{1}^{-1}$ for sample A can be reproduced well [solid line in Fig. 3(c)], yielding $E_{F}-E_{\mathrm{DP}} \sim-60 \mathrm{meV}$ [open square in Fig. 3(d)], roughly consistent with that estimated from the hole concentration and the band calculation (for details, see the Supplemental Material [41]). We also note that the orbital contribution to $T_{1}^{-1}$ was theoretically estimated to be at least an order of magnitude smaller than the observed spin contribution [46]. Possible enhancement of orbital contribution from that based on Ref. [46] was discussed [47]. The good scaling of $D(E)$ and $\left(T_{1} T\right)^{-1 / 2}$, however, indicates the orbital contribution can be neglected.

\section{E. Giant orbital diamagnetism of Dirac electrons}

Confirming that $\left(T_{1} T\right)^{-1 / 2}$ is a good measure of $D\left(E_{F}\right)$,we can estimate roughly the spin contribution $K_{\text {spin }}$ in the observed $K$. The Korringa relation, $T_{1} T K_{\text {spin }}{ }^{2}=S$, yields the linear dependence of $K_{\text {spin }}$ on $\left(T_{1} T\right)^{-1 / 2}$ with the slope $S^{1 / 2}$. For a simple metal with an isotropic Fermi surface, the Korringa value $S$ is determined by the gyromagnetic-ratio of the nuclei under observation, $\gamma_{\mathrm{n}}$, and the gyromagnetic-ratio of an electron, $\gamma_{e}$, as $S=\hbar / 4 \pi k_{B} \times\left(\gamma_{e} / \gamma_{n}\right)^{2}$. The $p$ dependence of the $g$ factor could modify the Korringa relationship as $K_{\text {spin }}$ is in proportion to $g$ [48]. The consistency of $K-\left(T_{1} T\right)^{-1 / 2}$ and $K-\chi$ relationships, which we will discuss below, however, indicates that the $p$ dependence of the $g$ factor should not be appreciable within the range of $p$ investigated here and that $g$ is unlikely to be strongly modified from 2 . The plot of $K$ as a function of $\left(T_{1} T\right)^{-1 / 2}$ is shown in Fig. 4(b). $K$ decreases with decreasing $\left(T_{1} T\right)^{-1 / 2}$, a measure of the density of states, from sample $\mathrm{E}$ to sample $\mathrm{A}$, much more rapidly than the expected linear relationship $K_{\text {spin }}=S^{1 / 2}\left(T_{1} T\right)^{-1 / 2}$ (gray dashed line). The strongly nonlinear decrease of $K$ from sample E can be naturally explained by the superposition of an additional orbital contribution $K_{\text {orb }}$, which increases rapidly with increasing $E_{F}$ (decreasing $p$ ) towards the Dirac mass gap.

It is known that the effective Korringa value $S^{*}$, inferred from an experimentally observed slope of $K-\left(T_{1} T\right)^{-1 / 2}$, is often larger than $S$ calculated from the gyromagnetic ratio by more than a factor of 2 even in a simple metal. The black dashed line with an enhanced $S^{*} \sim 6.3 S$ in Fig. 4(b) connects the data for all the heavily doped samples from $\mathrm{E}$ to $\mathrm{B}$. This assumes that $K$ is fully dominated by $K_{\text {spin }}\left(K_{\text {orb }} \sim 0\right)$ for these samples and therefore gives the upper-bound estimate for $K_{\text {spin }}$. Considering that the $E_{F}$ s of samples E-B are outside the Dirac band regime, the assumption of $K_{\text {orb }} \sim 0$ for them highly likely captures the reality better than the $S^{*}=S$ limit.
Even with the maximum estimate of $K_{\text {spin }}$, Fig. 4(b) clearly indicates that a large orbital contribution must be incorporated to account for the enhanced diamagnetic $K$ for sample A.

Taking a closer look at Fig. 4(b), we recognize the two important features to identify the orbital and the spin contributions in the $K-\chi$ plot. First, within the Korringa relationship, the spin contribution in low- $p$ sample $\mathrm{A}$ is negligibly small as compared with those of the other samples. Second, the $K-\left(T_{1} T\right)^{-1 / 2}$ line for each sample, representing the correlation between the $T$ dependences of $K(T)$ and $\left(T_{1} T\right)^{-1 / 2}$ of a given sample, has a negative slope (dotted lines) for sample $C$ and $\mathrm{E}$ and an almost infinite slope for sample A. They do not follow at all the linear behavior with a positive slope expected from the Korringa relationship. This very likely implies the presence of an additional $T$-dependent contribution to $K(T)$ other than $K_{\text {spin }}$, which is small but dominates the slope of $K-\left(T_{1} T\right)^{-1 / 2}$ line for each sample and can be ascribed naturally to $K_{\text {orb }}(T)$.

Let us now return to the $K-\chi$ plot in Fig. 4(a) with the information from Fig. 4(b). As $K_{\text {spin }} \sim 0$, the $K-\chi$ relationship for sample A should represent that for the nonspin contributions, $K=K_{\text {chem }}+K_{\text {orb }}=K_{\text {chem }}+A_{\text {orb }} \chi_{\text {orb }}$, which is indicated by the red solid line in Fig. 4(a) and gives an estimate of $A_{\text {orb }}=88 \pm 14 \mathrm{kOe} / \mu_{B}$. We can ascribe the slopes of the $K-\chi$ lines of $83 \pm 9 \mathrm{kOe} / \mu_{B}$ for sample $\mathrm{C}$ and $68 \pm 22 \mathrm{kOe} / \mu_{B}$ for sample $\mathrm{E}$ (green and blue dashed lines) close to $A_{\text {orb }}=$ $88 \pm 14 \mathrm{kOe} / \mu_{B}$ to the small but finite orbital contribution, the weak $T$ dependence of $K_{\text {orb }}(T)$ and $\chi_{\text {orb }}(T)$. The predominance of the orbital contribution $K_{\text {orb }}(T)$ in the $T$ dependence of $K(T)$ can naturally account for the negative slopes of $K-\left(T_{1} T\right)^{-1 / 2}$ for each sample in Fig. 4(b). For samples other than sample A, the $T$ dependences of $K_{\text {orb }}(T)$ are weakly negative and those of $\left(T_{1} T\right)^{-1 / 2}$ are very weakly positive. For sample $\mathrm{A},\left(T_{1} T\right)^{-1 / 2} \sim 0$ and its $T$ dependence is negligible as compared with the other samples.

The almost parallel and upward shift of the $K-\chi$ lines for samples $\mathrm{C}$ and $\mathrm{E}$ from the nonspin contributions line, $K=$ $K_{\text {chem }}+A_{\text {orb }} \chi_{\text {orb }}$, in Fig. 4(a) should then represent the superposition of an additional spin contribution $K_{\text {spin }}=A_{\text {spin }} \chi_{\text {spin }}$. As $\chi_{\text {spin }}$ is positive, $A_{\text {spin }}$ should be positive and larger than $A_{\text {orb }}$. The positive sign strongly suggests that $A_{\text {spin }}$ is determined by an $s$-electron-like Fermi contact interaction, which may be induced to the conduction electrons in the $\mathrm{Pb} 6 p$ bands by an $s p$ hybridization or by a strong spin-orbit coupling of $\mathrm{Pb}$ [49] as discussed in ${ }^{209} \mathrm{Bi}$ NMR on half-Heusler compounds [50]. The magnitude of $A_{\text {spin }}$, however, can be determined only with arbitrariness without any further assumption. For any positive $A_{\text {spin }}$ larger than $A_{\text {orb }}$, one can choose $K_{\text {spin }}$ and $\chi_{\text {spin }}$ which satisfy $K_{\text {spin }}=A_{\text {spin }} \chi_{\text {spin }}$ and bring the $K-\chi$ lines for samples $\mathrm{C}$ and $\mathrm{E}$ onto the nonspin contributions line $K=K_{\text {chem }}+A_{\text {orb }}+\chi_{\text {orb }}$ (red line) with the shift of $-K_{\text {spin }}$ and $-\chi_{\text {spin }}$ (see also Fig. S5(a) [41]). The shifted $K-\chi$ points on the nonspin contributions line represent $K=K_{\text {chem }}+A_{\text {orb }} \chi_{\text {orb }}$ of each sample.

To further narrow down the choice of $A_{\text {spin }}, K_{\text {spin }}$ and $\chi_{\text {spin }}$, the assumption of $K_{\text {orb }}=A_{\text {orb }} \chi_{\text {orb }} \sim 0$ for samples $\mathrm{C}$ and $\mathrm{E}$ may be reasonable, as their Fermi levels are located outside the Dirac bands. Then the $K-\chi$ relationship over samples $\mathrm{C}$ and $\mathrm{E}$ should constitute a universal nonorbital contributions line, $K=K_{\text {chem }}+A_{\text {spin }} \chi_{\text {spin }}$, neglecting the small 
temperature dependence of $K$ and $\chi$. The crossing point between the nonorbital contributions line and the nonspin line $K=K_{\text {chem }}+A_{\text {orb }} \chi_{\text {orb }}$ [red line in Fig. 4(a)] corresponds to $K_{\text {chem }}$ and the corresponding offset susceptibility $\chi_{0}(=$ $\left.\chi-\chi_{\text {spin }}-\chi_{\text {orb }}\right)$. The nonorbital contributions line $K=K_{\text {chem }}+$ $A_{\text {spin }} \chi_{\text {spin }}$ with the assumption of $K_{\text {orb }}=0$ for samples $\mathrm{C}$ and $\mathrm{E}$ can be roughly drawn as the black dashed line in Fig. 4(a). This nonorbital line and the crossing point with the nonspin line in Fig. 4(a) yields estimates of $A_{\text {spin }}=$ $210 \mathrm{kOe} / \mu_{B}, K_{\text {chem }}=0.055 \%$ (gray horizontal line), $\chi_{0}=$ $-8.1 \times 10^{-5} \mathrm{emu} / \mathrm{mol}$ (gray vertical line), and $K_{\text {spin }} \sim 0.042 \%$ and $\sim 0.102 \%$ for samples $\mathrm{C}$ and $\mathrm{E}$, respectively (blue vertical arrows). As shown in Fig. 4(b), the estimated $K_{\text {spin }}$ (blue vertical arrows) and $K_{\text {chem }}$ (horizontal line) from Fig. 4(a) are fully consistent with those estimated from the Korringa relation with $S^{*}=6.3 S$ where $K_{\text {orb }}=0$ is also assumed. The $\chi_{0}$ obtained above represents the core contribution to $\chi$ and agrees well with the core susceptibility of $-8.5 \times 10^{-5} \mathrm{emu} / \mathrm{mol}$ estimated from the atomic values in the literature [39], which justifies the assumption of $K_{\text {orb }}=0$ as the first approximation.

Using the $K_{\text {chem }}$ and the $\chi_{0}$ consistently determined for $K-\chi$ and $K-\left(T_{1} T\right)^{-1 / 2}$ in Figs. 4(a) and 4(b), a large diamagnetic orbital contribution in the Knight shift $K$ and the bulk magnetic susceptibility $\chi, K_{\text {orb }}=-0.09 \%$ and $\chi_{\text {orb }}=$ $-5.4 \times 10^{-5} \mathrm{emu} / \mathrm{mol}$ at $70 \mathrm{~K}$ and $K_{\text {orb }}=-0.06 \%$ and $\chi_{\text {orb }}=$ $-3.6 \times 10^{-5} \mathrm{emu} / \mathrm{mol}$ at $300 \mathrm{~K}$, are estimated for sample A. These orbital contributions apparently dominate the distinct diamagnetism in sample A of which Fermi level lies in the Dirac bands. The distinct behavior of $K$ and $T_{1}^{-1}$ in sample A was also observed in other two samples (see Fig. S1 [41]), which confirms the reproducibility of our results.

The estimated $A_{\text {orb }} \sim 88 \mathrm{kOe} / \mu_{B}$ from the $K-\chi$ plot implies the unconventional character of giant orbital diamagnetism. $K_{\text {orb }}$ is normally driven by a van Vleck paramagnetic susceptibility with $A_{\text {orb }}=2\left\langle r^{-3}\right\rangle$ [51] determined by the distance $r$ between the nuclei and the orbiting electrons. $A_{\text {orb }} \sim$ $2000 \mathrm{kOe} / \mu_{B}$ is estimated for $6 p$ orbitals of $\mathrm{Pb}$ [52], which is one order of magnitude larger than the experimentally observed $A_{\text {orb }} \sim 88 \mathrm{kOe} / \mu_{B}$ for the Dirac semimetal $\mathrm{Sr}_{3} \mathrm{PbO}$. The hybridization of $\mathrm{Pb} 6 p$ Dirac holes with $\mathrm{Sr} 4 d$ states and other orbital states could reduce the calculated $A_{\text {orb }}$ but not an order of magnitude. The small $A_{\text {orb }} \sim 88 \mathrm{kOe} / \mu_{B}$ therefore implies that the orbiting of spatially spread itinerant electrons, not of those completely confined within the atomic orbitals, is in charge of the observed large orbital diamagnetism. If Dirac electrons were uniform free electron gas and not confined in the atomic orbital at all, on the other hand, we would have an estimate of $A_{\text {orb }}<1 \mathrm{kOe} / \mu_{B}$ [53], orders of magnitude smaller than the experimentally observed $A_{\text {orb }} \sim 88 \mathrm{kOe} / \mu_{B}$. The drastic enhancement from the free electron estimate is reasonable as the Dirac electrons in $\mathrm{Sr}_{3} \mathrm{PbO}$ are not completely free from the atomic orbital and hopping from one atomic orbital to the others. These comparisons are fully consistent with the theoretical picture of giant orbital diamagnetism based on the interband mixing of itinerant Dirac electrons on the crystal lattice, which is distinct from the conventional orbital magnetism of the Van Vleck type. The hyperfine coupling from the orbiting of itinerant electrons has not been understood on the microscopic level yet, which at

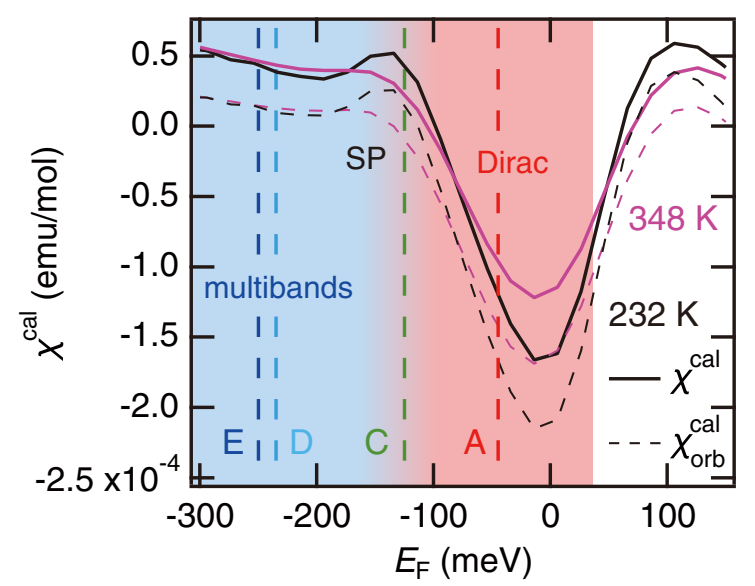

FIG. 5. Theoretical calculation of magnetic susceptibility as a function of the Fermi level $E_{F}$ for $\mathrm{Sr}_{3} \mathrm{PbO}$ antiperovskite. The Fermi level $E_{F}$ dependence of magnetic susceptibility $\chi^{\mathrm{cal}}$ at $232 \mathrm{~K}(0.2 \mathrm{eV})$ and $348 \mathrm{~K}(0.3 \mathrm{eV})$ (solid lines), calculated for tight-binding bands of $\mathrm{Sr}_{3} \mathrm{PbO}$ by incorporating the interband effect. The diamagnetic contributions from the core electrons $\chi_{0}$, which are temperature and hole density independent constants, are not included in the calculation. To compare with the experimental results, $\chi^{\text {cal }}+\chi_{0}$ should be used. A large diamagnetism grows with approaching the Dirac mass gap. The deconvoluted orbital contribution $\chi_{\text {orb }}^{\text {cal }}$ at 232 and $348 \mathrm{~K}$ are shown by the dashed lines. The experimentally determined $E_{F}$ for samples A-E are indicated by the vertical dashed lines.

this point does not allow us a more quantitative estimate of $A_{\text {orb }}$ to compare with the experimental result.

\section{F. Comparison with theoretical calculation}

The hole-concentration $p$ (hence Fermi level $E_{F}$ ) dependence of the magnetic susceptibility $\chi(T)$ in Fig. 2(f) and the predominance of the orbital contribution in the enhanced diamagnetism are reproduced by a theoretical calculation of the magnetic susceptibility $\chi^{\text {cal }}$ based on the expression in Eqs. (3)-(6), which explicitly includes the interband effects. Figure 5 indicates $\chi^{\text {cal }}$ (solid lines) and its deconvoluted orbital component $\chi^{\text {cal }}$ orb (dashed lines) as a function of $E_{F}$ at $T=232 \mathrm{~K}(0.2 \mathrm{eV})$ and $348 \mathrm{~K}(0.3 \mathrm{eV})$, calculated for the tight-binding bands of $\mathrm{Sr}_{3} \mathrm{PbO}$ [27]. Note that the calculated $\chi^{\text {cal }}$ does not include the contribution from the core electrons $\chi_{0}$, a $p$ - and $T$-independent constant, and therefore represents $\chi-\chi_{0}$. An enhanced diamagnetism in $\chi^{\mathrm{cal}}$ shows up when $E_{F}$ is in the Dirac band. Apparently, the orbital component $\chi^{\text {cal }}{ }_{\text {orb }}$ dominates the enhanced diamagnetism. When $E_{F}$ lies below the Dirac band regime, the calculated $\chi^{\mathrm{cal}}$ orb is much smaller than that in the Dirac band regime, which justifies the assumption of $K_{\text {orb }} \sim 0$ for samples C and E. A small but appreciable $T$ dependence of $\chi^{\text {cal }}$ is seen particularly in and near the Dirac band regime in Fig. 5, which originates from the orbital contribution $\chi_{\text {orb }}^{\text {cal }}(T)$ and changes sign from positive to negative upon going away from the mass gap. This is consistent with the increase and the decrease of experimental $\chi_{\mathrm{orb}}(T)$ and $\chi(T)$ with increasing $T$ for sample A $\left(E_{F}=-45 \mathrm{meV}\right)$ and sample $\mathrm{C}\left(E_{F}=-125 \mathrm{meV}\right)$, respectively. These qualitative agreements between the theory and the experiment provide a 
further support for the validity of the above analysis of NMR results.

Quantitatively, however, the calculation based on the tightbinding model does not allow us to capture the details of experimental results. $\chi^{\text {cal }}$ for $E_{F}=-45 \mathrm{meV}$ (corresponding to sample A) shows an additional diamagnetic contribution of $\sim-2 \times 10^{-4} \mathrm{emu} / \mathrm{mol}$ (essentially orbital in origin) as compared to those for $E_{F}<-100 \mathrm{meV}$ (samples B-E). This is almost a factor of four larger than the experimental orbital contribution $\chi_{\text {orb }} \sim 0.5 \times 10^{-4} \mathrm{emu} / \mathrm{mol}$ for sample A, which is difficult to account for only by the strong $E_{F}$ dependence of $\chi^{\text {cal }}$ and the ambiguity in the estimate of $E_{F} \cdot \chi^{\text {cal }}$ orb and $\chi^{\mathrm{cal}}$ appear to be overestimated within the framework of the present calculation.

\section{CONCLUSION}

In conclusion, our ${ }^{207} \mathrm{~Pb}$ NMR study of the 3D Dirac electron system $\mathrm{Sr}_{3} \mathrm{PbO}$ antiperovskite clearly revealed the orbital origin of large diamagnetism observed in the bulk magnetic susceptibility when its $E_{F}$ lies in the Dirac bands. This orbital diamagnetism is distinct from the ordinary orbital magnetism in that the orbiting electrons are not confined within the atomic orbitals but hop between the atomic orbitals. These observations are fully consistent with the microscopic picture of giant orbital diamagnetism of Dirac electrons established theoretically after the debates over decades and provide firm experimental evidence of such. The calculated orbital susceptibilities as a function of $E_{F}$ and $T$, based on the theories, indeed reproduce qualitatively the experimentally isolated orbital contribution to the magnetic susceptibility. Our results open up a fascinating possibility to further explore not only the intraband effects but also the interband effects in topological semimetals.

\section{ACKNOWLEDGMENTS}

We thank D. Hwang, O. Sushkov, T. Hirosawa, and H. Maebashi for fruitful discussions and comments, and K. Pflaum for technical assistance. Part of the computations in this work has been done using the facilities of the Supercomputer Center, the Institute for Solid State Physics, the University of Tokyo. This work was supported by Japan Society for the Promotion of Science (JSPS) KAKENHI (Grants No. 24224010, No. 15K13523, No. JP15H05852, No. JP15K21717, No. 17H01140, No. 18H01162, and No. 17J05243), JSPS Core-to-Core Program (A) Advanced Research Networks, and the Alexander von Humboldt Foundation. S.S. acknowledges financial support by JSPS and the Materials Education program for the future leaders in Research, Industry, and Technology (MERIT).
[1] S. M. Young, S. Zaheer, J. C. Y. Teo, C. L. Kane, E. J. Mele, and A. M. Rappe, Dirac Semimetal in Three Dimensions, Phys. Rev. Lett. 108, 140405 (2012).

[2] B.-J. Yang and N. Nagaosa, Classification of stable threedimensional Dirac semimetals with nontrivial topology, Nat. Commun. 5, 4898 (2014).

[3] S.-Y. Xu, C. Liu, S. K. Kushwaha, R. Sankar, J. W. Krizan, I. Belopolski, M. Neupane, G. Bian, N. Alidoust, T.-R. Chang, H.T. Jeng, C.-Y. Huang, W.-F. Tsai, H. Lin, P. P. Shibayev, F.-C. Chou, R. J. Cava, and M. Z. Hasan, Observation of Fermi arc surface states in a topological metal, Science 347, 294 (2015).

[4] L. P. He, X. C. Hong, J. K. Dong, J. Pan, Z. Zhang, J. Zhang, and S. Y. Li, Quantum Transport Evidence for the ThreeDimensional Dirac Semimetal Phase in $\mathrm{Cd}_{3} \mathrm{As}_{2}$, Phys. Rev. Lett. 113, 246402 (2014).

[5] A. Narayanan, M. D. Watson, S. F. Blake, N. Bruyant, L. Drigo, Y. L. Chen, D. Prabhakaran, B. Yan, C. Felser, T. Kong, P. C. Canfield, and A. I. Coldea, Linear Magnetoresistance Caused by Mobility Fluctuations in N-Doped $\mathrm{Cd}_{3} \mathrm{As}_{2}$, Phys. Rev. Lett. 114, 117201 (2015).

[6] J. Xiong, S. K. Kushwaha, T. Liang, J. W. Krizan, M. Hirschberger, W. Wang, R. Cava, and N. Ong, Evidence for the chiral anomaly in the Dirac semimetal $\mathrm{Na}_{3} \mathrm{Bi}$, Science 350, 413 (2015).

[7] Q. Li, D. E. Kharzeev, C. Zhang, Y. Huang, I. Pletikosić, A. Fedorov, R. Zhong, J. Schneeloch, G. Gu, and T. Valla, Chiral magnetic effect in $\mathrm{ZrTe}_{5}$, Nat. Phys. 12, 550 (2016).

[8] M. Ogata and H. Fukuyama, Orbital magnetism of Bloch electrons I. General formula, J. Phys. Soc. Jpn. 84, 124708 (2015).

[9] H. Fukuyama and R. Kubo, Interband effects on magnetic sus- ceptibility. II. Diamagnetism of bismuth, J. Phys. Soc. Jpn. 28, 570 (1970).

[10] Y. Fuseya, M. Ogata, and H. Fukuyama, Transport properties and diamagnetism of Dirac electrons in bismuth, J. Phys. Soc. Jpn. 84, 012001 (2014).

[11] A. Goetz and A. B. Focke, The crystaldiamagnetism of bismuth crystals, Phys. Rev. 45, 170 (1934).

[12] D. Shoenberg and M. Z. Uddin, The magnetic properties of bismuth. i. dependence of susceptibility on temperature and addition of other elements, Proc R. Soc. London Ser. A 156, 687 (1936).

[13] L. Wehrli, Die magnetische suszeptibilität von bi und Bi-Sblegierungen, Phys. Kondens. Mater. 8, 87 (1968).

[14] B. Lenoir, M. Cassart, J.-P. Michenaud, H. Scherrer, and S. Scherrer, Transport properties of Bi-rich Bi-Sb alloys, J. Phys. Chem. Solids. 57, 89 (1996).

[15] F. Orbanić, M. Novak, M. Baćani, and I. Kokanović, Quantum oscillations in a lead chalcogenide three-dimensional Dirac system, Phys. Rev. B. 95, 035208 (2017).

[16] A. Pariari and P. Mandal, Coexistence of topological Dirac fermions on the surface and three-dimensional Dirac cone state in the bulk of $\mathrm{ZrTe}_{5}$ single crystal, Sci. Rep. 7, 40327 (2017).

[17] N. L. Nair, P. T. Dumitrescu, S. Channa, S. M. Griffin, J. B. Neaton, A. C. Potter, and J. G. Analytis, Thermodynamic signature of Dirac electrons across a possible topological transition in $\mathrm{ZrTe}_{5}$, Phys. Rev. B. 97, 041111(R) (2018).

[18] A. W. Rost, J. Kim, S. Suetsugu, V. Abdolazimi, K. Hayama, J. Bruin, C. Mühle, K. Kitagawa, A. Yaresko, J. Nuss, and H. Takagi, Inverse-perovskites $A_{3} B \mathrm{O}(A=\mathrm{Sr}, \mathrm{Ca}, \mathrm{Eu} / B=\mathrm{Pb}$, 
Sn): A platform for control of Dirac and Weyl fermions, APL Mater. 7, 121114 (2019).

[19] B. F. Williams and R. R. Hewitt, Nuclear magnetic resonance in bismuth metal, Phys. Rev. 146, 286 (1966).

[20] F. N. Gygax, A. Schenck, A. J. van der Wal, and S. Barth, Higher-Order Angular Dependence of the Positive-Muon Knight Shift in Bismuth, Phys. Rev. Lett. 56, 2842 (1986).

[21] E. Lippelt, P. Birrer, F. Gygax, B. Hitti, A. Schenck, and M. Weber, Diamagnetism and muon Knight shift in semiconducting $\mathrm{Bi}_{1-x} \mathrm{Sb}_{x}$ single crystals, Z. Phys. B Condens. Mater. 86, 367 (1992).

[22] A. Berger, H. Bertschat, H.-E. Mahnke, B. Spellmeyer, and W. Shen, Knight shift of sp-elements in bismuth, Hyperfine Interact. 34, 547 (1987).

[23] W. Shen, A. Berger, H. Bertschat, H.-E. Mahnke, and B. Spellmeyer, Strong local diamagnetism in bismuth, Phys. Lett. A. 125, 489 (1987).

[24] W. A. MacFarlane, C. B. L. Tschense, T. Buck, K. H. Chow, D. L. Cortie, A. N. Hariwal, R. F. Kiefl, D. Koumoulis, C. D. P. Levy, I. McKenzie, F. H. McGee, G. D. Morris, M. R. Pearson, Q. Song, D. Wang, Y. S. Hor, and R. J. Cava, $\beta$-detected NMR of ${ }^{8} \mathrm{Li}^{+}$in $\mathrm{Bi}, \mathrm{Sb}$, and the topological insulator $\mathrm{Bi}_{0.9} \mathrm{Sb}_{0.1}$, Phys. Rev. B. 90, 214422 (2014).

[25] J. Nuss, C. Mühle, K. Hayama, V. Abdolazimi, and H. Takagi, Tilting structures in inverse perovskites, $M_{3} T t \mathrm{O}(M=\mathrm{Ca}, \mathrm{Sr}$, $\mathrm{Ba}, \mathrm{Eu} ; T t=\mathrm{Si}, \mathrm{Ge}, \mathrm{Sn}, \mathrm{Pb})$, Acta Crystallogr. Sec. B 71, 300 (2015).

[26] T. Kariyado and M. Ogata, Three-dimensional Dirac electrons at the Fermi energy in cubic inverse perovskites: $\mathrm{Ca}_{3} \mathrm{PbO}$ and its family, J. Phys. Soc. Jpn. 80, 083704 (2011).

[27] T. Kariyado and M. Ogata, Low-energy effective Hamiltonian and the surface states of $\mathrm{Ca}_{3} \mathrm{PbO}$, J. Phys. Soc. Jpn. 81, 064701 (2012)

[28] T. H. Hsieh, J. Liu, and L. Fu, Topological crystalline insulators and Dirac octets in antiperovskites, Phys. Rev. B. 90, 081112(R) (2014).

[29] C.-K. Chiu, Y.-H. Chan, X. Li, Y. Nohara, and A. P. Schnyder, Type-II Dirac surface states in topological crystalline insulators, Phys. Rev. B. 95, 035151 (2017).

[30] S. Suetsugu, K. Hayama, A. W. Rost, J. Nuss, C. Mühle, J. Kim, K. Kitagawa, and H. Takagi, Magnetotransport in $\mathrm{Sr}_{3} \mathrm{PbO}$ antiperovskite, Phys. Rev. B. 98, 115203 (2018).

[31] Y. Obata, R. Yukawa, K. Horiba, H. Kumigashira, Y. Toda, S. Matsuishi, and H. Hosono, ARPES studies of the inverse perovskite $\mathrm{Ca}_{3} \mathrm{PbO}$ : Experimental confirmation of a candidate 3D Dirac fermion system, Phys. Rev. B. 96, 155109 (2017).

[32] R. K. Harris E. D. Becker, S. M. C. de Menezes, P. Granger, R. E. Hoffman, and K. W. Zilm, Further conventions for NMR shielding and chemical shifts (IUPAC Recommendations 2008), Pure Appl. Chem. 80, 59 (2008).

[33] P. Giannozzi, S. Baroni, N. Bonini, M. Calandra, R. Car, C. Cavazzoni, D. Ceresoli, G. L. Chiarotti, M. Cococcioni, I. Dabo, A. Dal Corso, S. Fabris, G. Fratesi, S. de Gironcoli, R. Gebauer, U. Gerstmann, C. Gougoussis, A. Kokalj, M. Lazzeri, L. Martin-Samos, N. Marzari, F. Mauri, R. Mazzarello, S. Paolini, A. Pasquarello, L. Paulatto, C. Sbraccia, S. Scandolo, G. Sclauzero, A. P. Seitsonen, A. Smogunov, P. Umari, and R. M. Wentzcovitch, QUANTUM ESPRESSO: A modular and open- source software project for quantum simulations of materials, J. Phys.: Condens. Matter 21, 395502 (2009).

[34] P. Giannozzi, O. Andreussi, T. Brumme, O. Bunau, M. Buongiorno Nardelli, M. Calandra, R. Car, C. Cavazzoni, D. Ceresoli, M. Cococcioni, N. Colonna, I. Carnimeo, A. Dal Corso, S. de Gironcoli, P. Delugas, R. A. DiStasio Jr, A. Ferretti, A. Floris, G. Fratesi, G. Fugallo, R. Gebauer, U. Gerstmann, F. Giustino, T. Gorni, J Jia, M. Kawamura, H.-Y. Ko, A. Kokalj, E. Küçükbenli, M. Lazzeri, M. Marsili, N. Marzari, F. Mauri, N. L. Nguyen, H.-V. Nguyen, A. Otero-de-la-Roza, L. Paulatto, S. Poncé, D. Rocca, R. Sabatini, B. Santra, M. Schlipf, A. P. Seitsonen, A. Smogunov, I. Timrov, T. Thonhauser, P. Umari, N. Vast, X. Wu, and S. Baroni, Advanced capabilities for materials modelling with QUANTUM ESPRESSO, J. Phys.: Condens. Matter 29, 465901 (2017).

[35] A. A. Mostofi, J. R. Yates, G. Pizzi, Y.-S. Lee, I. Souza, D. Vanderbilt, and N. Marzari, An updated version of wannier90: A tool for obtaining maximally-localised Wannier functions, Comput. Phys. Commun. 185, 2309 (2014).

[36] K.-T. Chen and P. A. Lee, Unified formalism for calculating polarization, magnetization, and more in a periodic insulator, Phys. Rev. B 84, 205137 (2011).

[37] G. Gómez-Santos and T. Stauber, Measurable Lattice Effects on the Charge and Magnetic Response in Graphene, Phys. Rev. Lett. 106, 045504 (2011).

[38] A. Raoux, F. Piéchon, J.-N. Fuchs, and G. Montambaux, Orbital magnetism in coupled-bands models, Phys. Rev. B 91, 085120 (2015).

[39] R. R. Gupta, Diamagnetic Susceptibility, Landolt-Börnstein New Series, Group II, Vol. 16 (Springer, Berlin, 1986).

[40] G. C. Carter, L. H. Bennett, and D. J. Kahan, Metallic shifts in NMR, Progress in Materials Science, Vol. 20 (Pergamon Press, Oxford, 1977).

[41] See Supplemental Material at http://link.aps.org/supplemental/ 10.1103/PhysRevB.103.115117 for details on theoretical calculation, further analysis, and additional graphs for different samples described in the main text.

[42] K. Miyagawa, M. Hirayama, M. Tamura, and K. Kanoda, ${ }^{13} \mathrm{C}$ NMR study on zero-gap state in the organic conductor $\theta$-(BEDT-TTF $)_{2} \mathrm{I}_{3}$ under pressure, J. Phys. Soc. Jpn. 79, 063703 (2010).

[43] H. Yasuoka, T. Kubo, Y. Kishimoto, D. Kasinathan, M. Schmidt, B. Yan, Y. Zhang, H. Tou, C. Felser, A. P. Mackenzie, and M. Baenitz, Emergent Weyl Fermion Excitations in TaP Explored by ${ }^{181}$ Ta Quadrupole Resonance, Phys. Rev. Lett. 118, 236403 (2017).

[44] S. Kitagawa, K. Ishida, M. Oudah, J. N. Hausmann, A. Ikeda, S. Yonezawa, and Y. Maeno, Normal-state properties of the antiperovskite oxide $\mathrm{Sr}_{3-x} \mathrm{SnO}$ revealed by ${ }^{119} \mathrm{Sn}-\mathrm{NMR}$, Phys. Rev. B 98, 100503(R) (2018).

[45] C. G. Wang, Y. Honjo, L. X. Zhao, G. F. Chen, K. Matano, R. Zhou, and G.-q. Zheng, Landau diamagnetism and Weylfermion excitations in TaAs revealed by ${ }^{75}$ As NMR and NQR, Phys Rev. B 101, 241110(R) (2020).

[46] T. Hirosawa, H. Maebashi, and M. Ogata, Nuclear spin relaxation time due to the orbital currents in Dirac electron systems, J. Phys. Soc. Jpn. 86, 063705 (2017).

[47] Z. Okvátovity, H. Yasuoka, M. Baenitz, F. Simon, and B. Dóra, Nuclear spin-lattice relaxation time in $\mathrm{TaP}$ and the 
Knight shift of Weyl semimetals, Phys. Rev. B 99, 115107 (2019).

[48] Y. Yafet, Hyperfine interaction due to orbital magnetic moment of electrons with large g factors, J. Phys. Chem. Solids 21, 99 (1961).

[49] L. Tterlikkis, S. D. Mahanti, and T. P. Das, Theory of the Knight shift and the relaxation time in lead, Phys. Rev. B. 1, 2041 (1970).

[50] X. Zhang, Z. Hou, Y. Wang, G. Xu, C. Shi, E. Liu, X. Xi, W. Wang, G. Wu, and X.-x. Zhang, NMR evidence for the topolog- ically nontrivial nature in a family of half-Heusler compounds, Sci. Rep. 6, 23172 (2016).

[51] A. Abragam, The Principles of Nuclear Magnetism (Clarendon Press, Oxford, 1961).

[52] A. Lurio and D. A. Landman, Hyperfine structure of the $(6 p)^{2}$ configuration of ${ }^{207} \mathrm{~Pb}$, J. Opt. Soc. A 60, 759 (1970).

[53] H. Maebashi, T. Hirosawa, M. Ogata, and H. Fukuyama, Nuclear magnetic relaxation and Knight shift due to orbital interaction in Dirac electron systems, J. Phys. Chem. Solids 128, 138 (2019). 